\title{
EXPERIMENTS AND SIMULATIONS OF MAXIMAL SCULLING PROPULSION: VORTICITY IMPULSE IN HUMAN BIOMECHANICS
}

ARTICLE · FEBRUARY 2016

READS

44

6 AUTHORS, INCLUDING:

Jose Manuel Redondo

Polytechnic University of Catalonia

247 PUBLICATIONS 1,102 CITATIONS

SEE PROFILE

Raul Arellano

University of Granada

129 PUBLICATIONS 317 CITATIONS

SEE PROFILE
Jackson Tellez Alvarez

Polytechnic University of Catalonia

25 PUBLICATIONS 10 CITATIONS

SEE PROFILE 


\title{
EXPERIMENTS AND SIMULATIONS OF MAXIMAL SCULLING PROPULSION: VORTICITY IMPULSE IN HUMAN BIOMECHANICS.
}

\author{
P. Furnanek ${ }^{1,2}$, J. M. Redondo ${ }^{2,3}$, A. Carrilloº ${ }^{2}$ J. Tellez $^{2}$, R. Arellano ${ }^{3}$ \\ and M. A. Sanchez ${ }^{2,3}$
}

\author{
${ }^{1}$ Department of Technical Mathematics, Faculty of Mechanical Engineering, Czech \\ Technical University in Prague, Karlovo nam. 13, 12135, CR. \\ 2 Department of Applied Physics, UPC-Barcelona Tech. Univ. Politecnica de Cataluña, \\ Campus Nord B5, 08034, Spain. \\ ${ }^{3}$ Faculty of Physical Activity and Sport Science, Univ.Granada. Cl Alfacar, Granada, \\ 18071, Spain.
}

\begin{abstract}
The detailed study of the turbulence and the fluid flow in sport is an open and exciting field of research, in particular in swimming and aquatic sports there is a wealth of new techniques that may aid performance. In swimming, thanks to measurement techniques like Particle Image Velocimetry (PIV), Particle Tracking (PT) or pattern analysis, now it is possible to measure the flow environment and not just the human movement. Numerical Computational Fluid Dynamics (CFD) is also a useful tool. We present several techniques stressing the importance of 3D effects and the dynamics of enhanced propulsion by hands and feet while the reduction in resistance need to be considered in an integrated way. Examples of Sculling, Hand wakes, Underwater Undulatory Swimming (UUS) and Vortex Filament Analysis (VFA) are all interesting to improve swimming techniques.
\end{abstract}

Keywords: Propulsion, Swimming, Arm Stroke, CFD, Turbulent wakes

\section{Introduction}

Swimming propulsion in humans is the result of the muscular force applied by the hands, arms and feet to the water and the aim in sport is to maximize effective propulsion minimizing energy and drag. Other aquatic sports like Sincronized Swimming or Waterpolo use the "Sculling” or "Egg beat" techniques against gravity. In fluid mechanics it is apparent that vorticity, defined as the curl of the velocity field in a flow has an important dynamical role, both in 2D and 3D [1]. Previous sport studies have recorded propulsive force during tethered swimming and used bubbles to trace the water flow (Arellano et al, 2002, 2006)[2,3]. One of the apparent conclusions of any type of flow visualization showed the appearance of vorticity in wakes. The importance was seen to be dominant in the best swimmers, whose circulation produced by both hands and feet (eddies or vortex structures) were more regular and more persistent [4]. Other direct measurements such as accelerometers or point Laser or Sonic flow velocity measurements are now widespread and may be used in Biomechanics in either Eulerian or Lagrangian set ups [5, 6]. As is common in swimming the arms and legs move in very complex fashion [7-10]. When non-steady motions occur produced by a swimmer, both Lift and Drag are just the components of the local motion. Kutta - Zhukovsky's condition is not met and unbound vortices are shed at the tips of the hands and feet in a turbulent 3D cascade forming a complex wake. The harmonic type of motions also need to consider added mass effects. In figure 1 showing the Granada swimming pool [2], we can see the asymmetry of the UUS motions. 
Studies in the animal world have also shown how vortices are generated during the flight of birds and the propulsion of fish or dolphins. [10,14], they show that there is a Strouhal number of about 0.2-0.3 associated to usual propulsion, which could be also associated to maximum efficiency. A line of micro bubbles is a good way to visualize the complex swimmer's pulling paths. Hub vortices are generated in screw type or propeller motions, and are observed in small propulsive movements of the hands performing sculling during synchronised swimming. Some specific sculling propulsion using only the arms are discussed below together with a comparison of Lift and Drag basic definitions used since Counsillman and others showed that lift was also important beside drag in human competitive swimming [13-16], the role of vorticity production and the control of wakes is much more important than previously thought $[4,11]$.

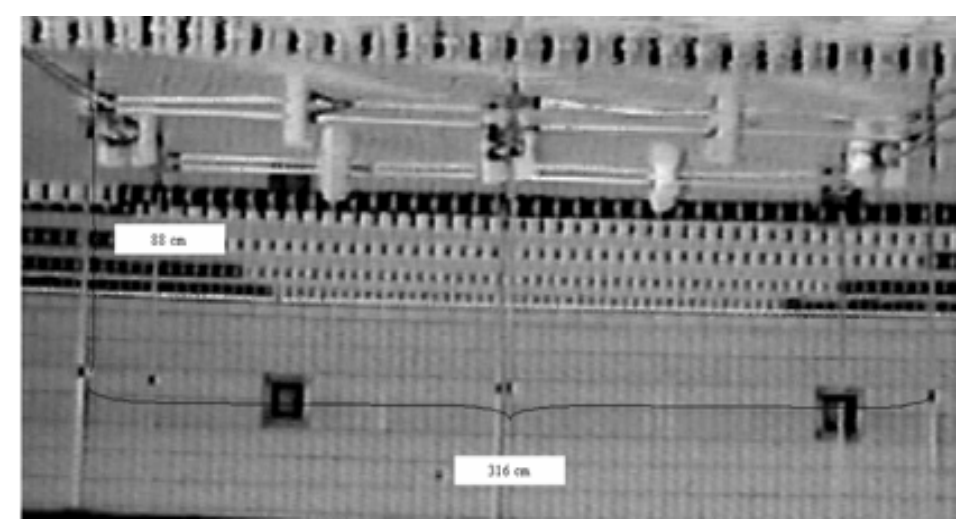

a)

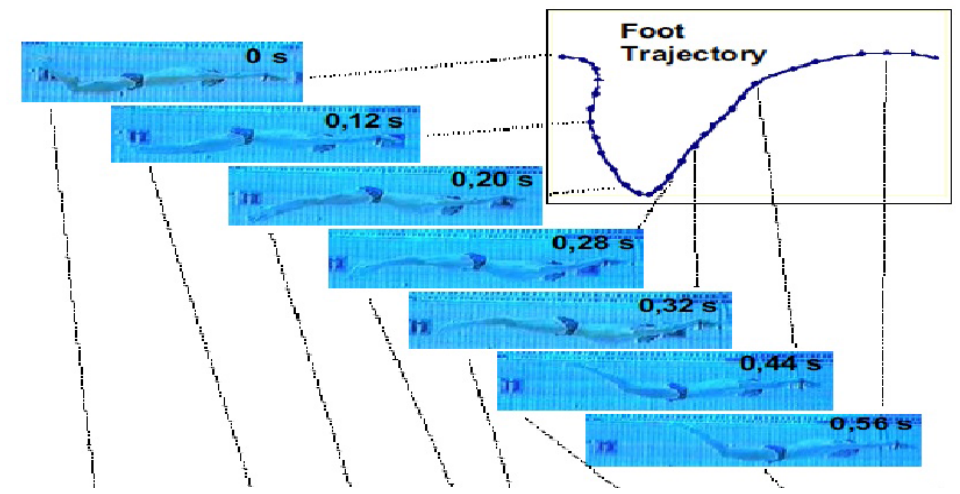

b)

Figure 1 Visualization of the development of vortex structures by movement of the feet in an instrumented swimming pool in Granada a) Redondo and Arellano(1998). Pattern of underwater undulatory kicking. (Arellano et al. 2006). Thanks to the different flow visualisation techniques vortices of different sizes are detected b).

We used different systems to observe vortices: Vortices generated during undulatory underwater swimming (UUS) and breaststroke-butterfly leg kicking injecting bubbles; Vortices produced by the hand in analytical situation in the lab using reflective small particles; Vortices created in the swimming pool and in real freestyle swimming and kicking using a bubble wall,... [2, 10]. 


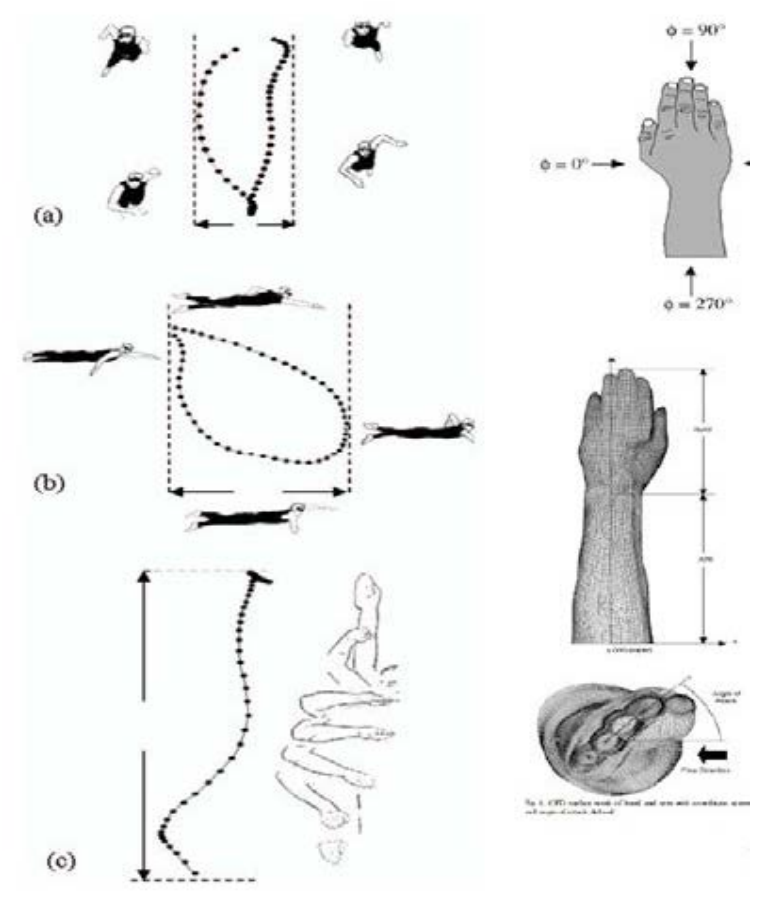

Figure 2 Hand Swimming patterns in competitive swimming [13].

Plastic tubes connected from an air compressor to the body of the swimmer with the exit at the toe or hand generate a bubble traces of the trajectory, easily observed during underwater body gliding. Without feet movement and during horizontal gliding, the small bubbles draw a line parallel to body displacement until they start rising due to buoyancy. PIV analysis of the flow, if the buoyancy of the air bubble array is small may give a 2D view of the flow. The use of a Laser sheet or more powerful light allows to improve the wake analysis It is important to have a 3D view of the basic coherent structures that are generated, because of the complexity of the flows. Seeding the pool with neutral buoyancy particles is the best solution. Observations show that the best swimmers generate bigger vortices at the end of the downward kick, and the production of vortex rings and lines is confirmed in all types of flows. The main vortex starts during the initial phase of the downward vertical movement, in the wake we found the vortex seems more coherent and rotates in the same place without displacement longer than for slower swimmers. Figure 2 shows the complex, average, motion of the hand.
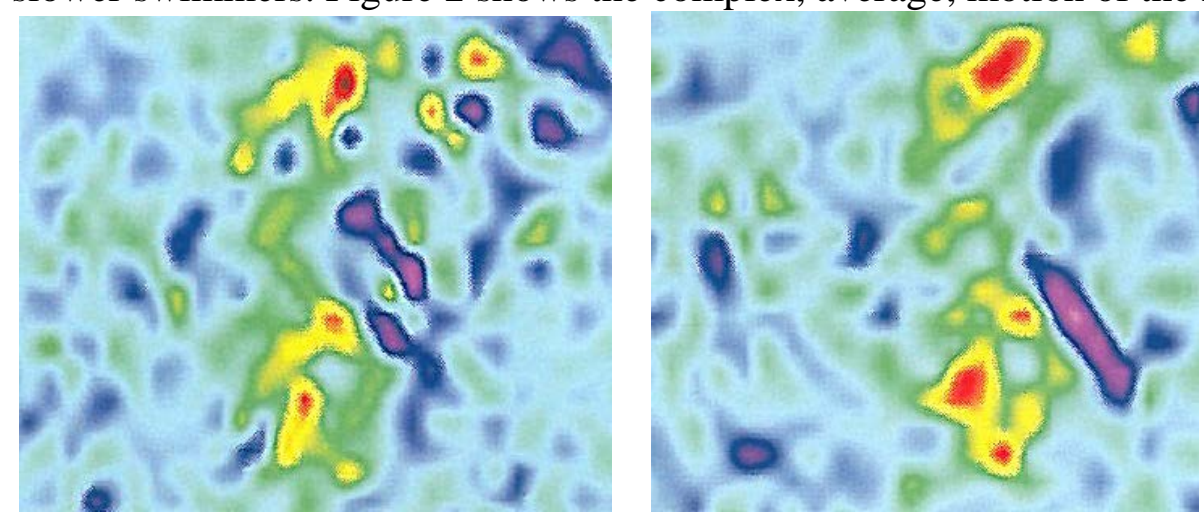

Figure 3. Example of vortex structures produced by a simulated UUS kick in a vertical central plane produced by a sudden plate movement. PIV with a Laser Sheet in the Laboratory. The red high positive vorticity and the blue negative values indicate the double toroidal shape of the vortex structure 


\section{Description of Vorticity Patterns and Propulsion}

A $2 \mathrm{~m}^{3}$ aquarium was utilised in the laboratory with small PIV 50 Micron particles were placed in the water with density similar to the brine. A $0.2 \mathrm{~W}$ green solid state laser projected a parallel plane of light produced by a cylindrical lens. The plane of Laser light allowed us to observe easily the position of the "Pliolite" resine particles. A video camera was placed perpendicular to the aquarium. The same system was set up in new experiments positioned in the middle of the pool lane (Fig 1) nearest to an underwater window. Trials and calibration points are necessary to capture the movement of hand crossing the seeded flow in the correct moment to show the vorticity structure by means of PIV using DigImage programme as shown in figure 3. Here the coherence of the vortex rotation after an UUS single kick shows a clear 3D double vortex ring of which the two red (dark-high vorticity) plane cuts indicate the generation of a large vortices during the stroke, which seem related to higher propulsion.

This effect for a single generation of a impulsive vortex ring has been analysed by Linden and Turner [17], also discussed in [4] but an statistical comparison of the momentum and vorticity in complex wakes, like in the measurements of lift and drag $[18,19]$ seems important to understand the role of coherent vorticity in propulsive wakes

The dimensionless Strouhal number for the UUS is the product of the peak-to-peak sum of the ventral and dorsal amplitudes of the toe $A=h_{-}+h_{+}$and the total kick frequency $f$ in relation to the total swimming speed $V$ and is related to the swimming efficiency [2-4, 14]. The Strouhal number defined as:

$$
S t=\frac{2 A \cdot f}{V}=\frac{2 A \cdot \tau}{T \cdot L}
$$

Which it may be interpreted as the inverse ratio of the forward swimming speed $V$ and the local vertical kick speed $w=2 \mathrm{~A} f$. As the kick induced vorticity may be estimated as twice the vortical induced ring $w$ velocity component divided by the size of the vortex ring ( $D$ becoming larger than $A$ ), which is soon organised according to well distinct Karman type cell pattern for the UUS, The critical factors that improve efficiency are related to the the secondary instability, and the final direction of the vorticity wake patterns. Best swimmers wake appearance seems more organised with lateral limitation of spanwise and streamwise vorticity components $\omega_{x, y}$. The Reynolds number based on the size of the swimmer $R e=\rho$ $L V / \mu$ increases, mostly due to the higher speed. The Strouhal number relationship with the swimming efficiency shows a maximum at about 0.3 . In order to compare different subjects, with a general hyperbolic function $y=1 / x$. The coordinates are:

$$
x=\frac{V \cdot f}{L}
$$

and

$$
y=\frac{A}{L}
$$


The parameter space $x(y)$ provides additional information to the efficiency Strouhal number dependence plot $\chi(S t)$. The basic theory of maximum propulsive efficiency for vortex rings with an aspect ratio of about $1 / 3=D / L$, also applies to vertical kicks of $A / L=1 / 3$. A simple way to envisage the observations is that high dinamical efficiency occurs when the speed of lateral vorticity generation is one third of the forward speed: $V=w$ St.
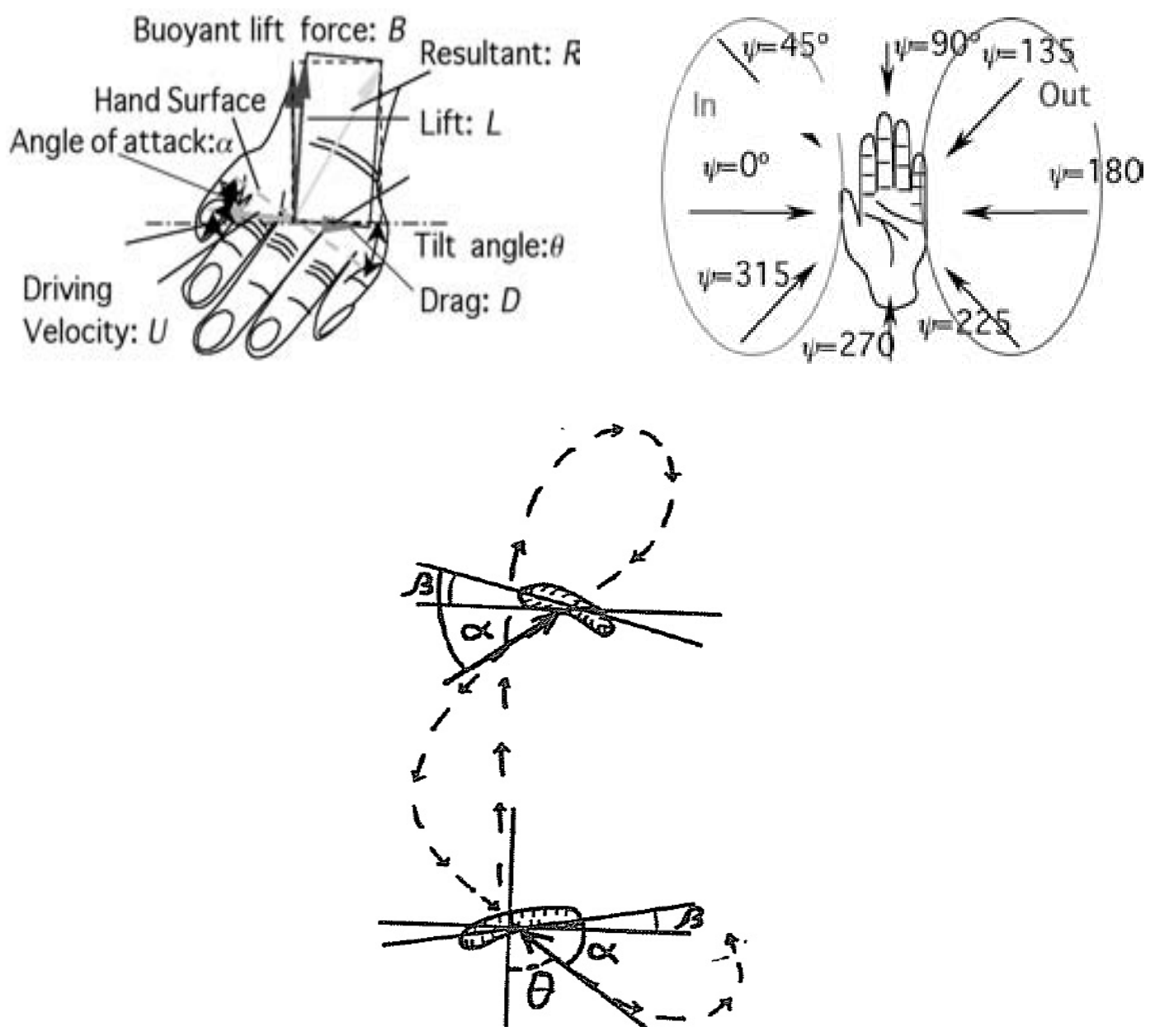

Figure 4. Different attack angles defined for a hand sweep considering wing or airfoil theory.The different lift and drag coefficients and hand tip patterns are a strong function of the variation of the Reynolds number as well as the tilt and azimuthal angle.[18-21]. 


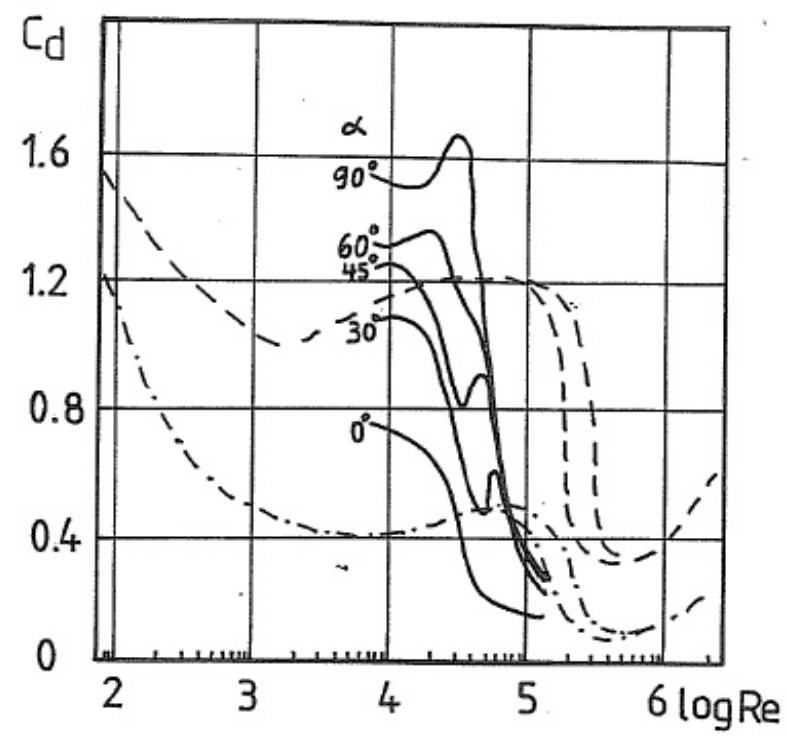

Figure 5. Variation of Drag coefficient with Reynolds number that depends on local vortex structures produced by the wakes of the hand, faster movements do not produce a quadratic increase [18].

\subsection{THE NATURAL VORTEX DESCRIPTORS}

The instantaneous iso-vorticity fields are first considered. During the the swimming wake spanwise vortex rings occurs, that progressively merge and generate the overall complex swimmer wake, including contributions from arms and legs. The complexity of all the local effects that aim to maximize propulsion and to minimize drag as well as energy needs to be analysed considering simpler effects.

The basic equations are the continuity and momentum equations, for an incompressible fluid the continuity equation reduces to

$$
\Delta \cdot u=0
$$

The equation of motion of an incompressible, homogeneous fluid with constant viscosity is:

$$
\frac{\partial u}{\partial t}+u_{i} \frac{\partial u}{\partial x_{i}}=-\frac{1}{\rho} \nabla p+v \nabla^{2} u
$$

where $p$ is the pressure, and $v$ kinematic viscosity. If the motion is primarily horizontal, then the flow is approximately two-dimensional, or at least we only measure in one plane, i.e.

$$
\mathrm{u}=(u, v, 0)
$$

where $u, v$ are the velocity components of a single plane independent of depth. In a strictly 2D flow of this form there is only one non-zero component of vorticity (the vertical) and

$$
\omega=(0,0, \omega)
$$

In the absence of dissipation due to viscosity, vorticity is conserved

$$
\frac{D \omega}{D t}=0
$$

And thus we may define a stream function $\psi$, so 


$$
\begin{aligned}
& u=\left(\frac{\partial \psi}{\partial y},-\frac{\partial \psi}{\partial x}, 0\right) \\
& \omega=-\nabla^{2} \psi
\end{aligned}
$$

And the equation of vorticity conservation becomes

$$
\frac{\partial \omega}{\partial t}+J(\varpi, \psi)=0
$$

In strictly 2D flows with no dissipation we have conservation of

$$
\begin{array}{ll}
\text { Energy } & \overline{u^{2}}=\int_{0}^{\infty} E(k, t) d k, \\
\text { Enstrophy } & \overline{\omega^{2}}=\int_{0}^{\infty} k^{2} E(k, t) d k
\end{array}
$$

Conservation of enstrophy results from conservation of vorticity $\omega$ (no stretching or twisting). In 2D flows but with weak dissipation the above results hold approximately. The streamwise vortex structures are normally much more fragmented even in near body positions. Spanwise vorticity is stronger due to the vertical human (and dolphin) kick. The vertical vorticity, though initially weak, lasts longer in swimmers wakes because of buoyancy and the role of the water-air surface.

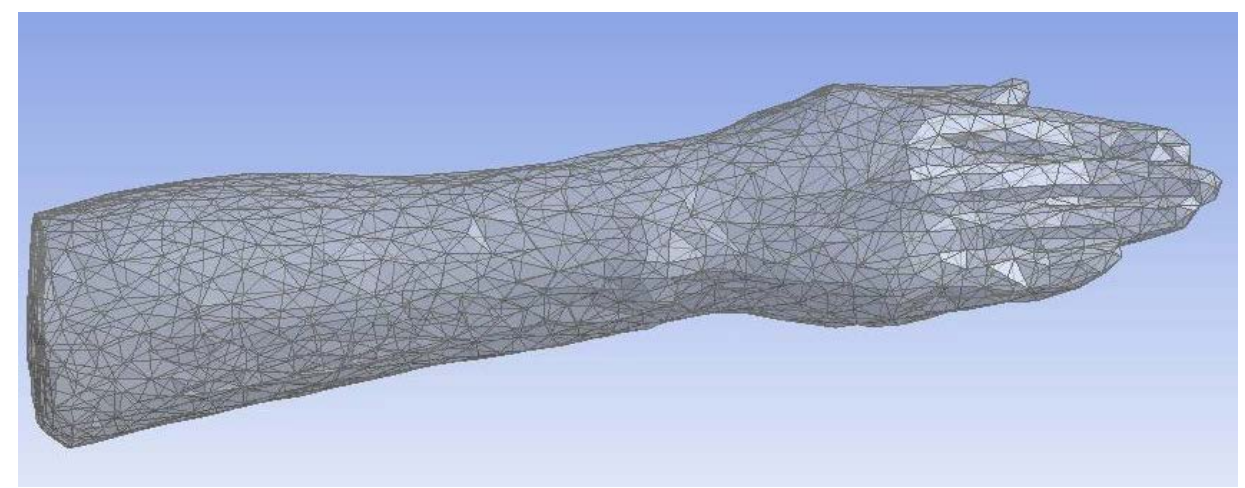

Figure 6. Mesh of a Hand model for Open Foam Drag simulations

In order to get a qualitative representation of the different propulsive structures shed by swimmers with a specific influence of the turbulent vorticity we propose a vertical plane plot of the parameter function $\omega(\psi)=-\nabla^{2} \psi(\psi)$ and its evolution measured using Correlation Image Velocimetry on the vorticity cores $S$ as:

$$
\frac{\partial(\rho S)}{\partial t}=-\operatorname{div}(\rho \mathbf{v} S)
$$




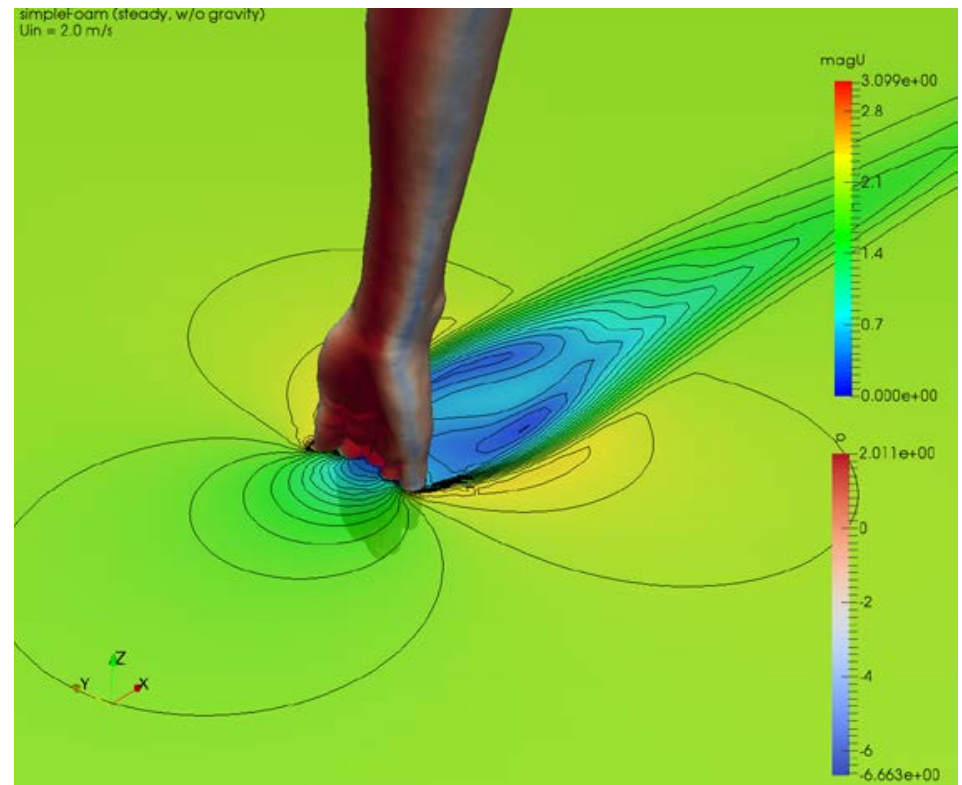

Figure 7. Hand wake at local velocity $2 \mathrm{~m} / \mathrm{s}$

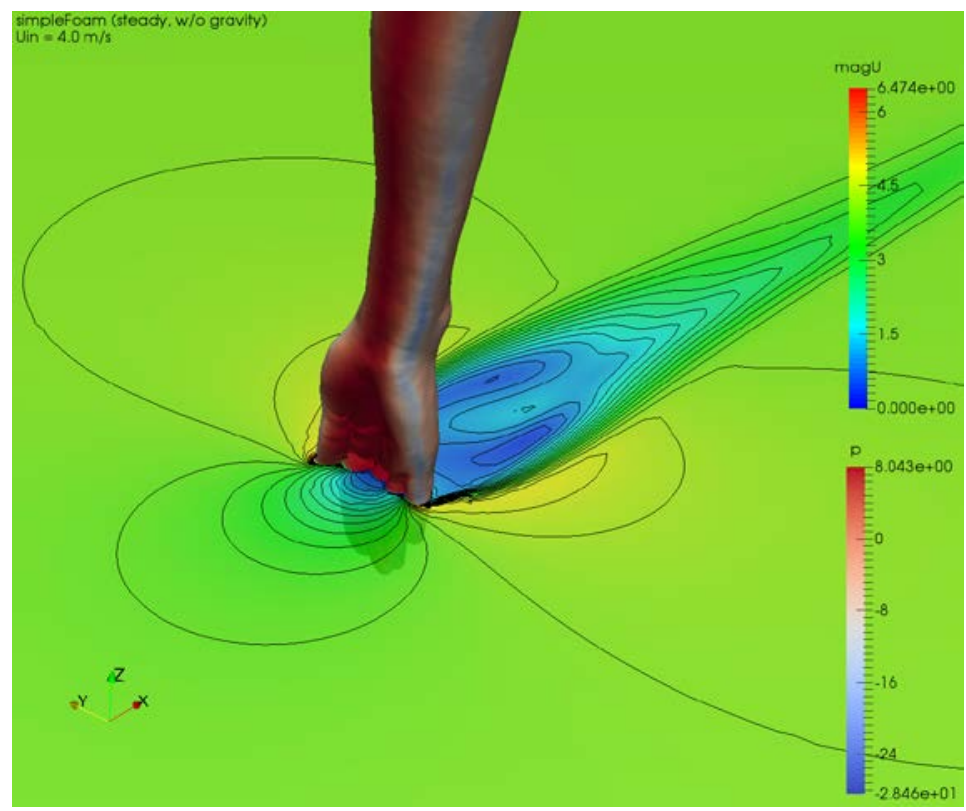

Figure 8. Hand wake at $4 \mathrm{~m} / \mathrm{s}$

\section{Drag and Lift in Hand Experiments and Numerical Simulations}

Different researchers [13-21] also found relationships between the complex patterns of the hands in swimming and vorticity formation controlled by the lift and drag interplay during non-steady motions. Observations and numerical simulations have improved markedly in recent years, but it is now necessary to quantify and classify, at least in statistical terms turbulent descriptors related to propulsive efficiency, for example the shear length-scale at a certain distance from the hand wake ( or the kick in UUS as discussed above). A possible definition is $L S i=V /\left(d V / d x_{i}\right)_{\max }$ for local wake profiles from hand, feet or the whole body ([18-22]). For microstructure measurements, either from micro probes of velocity or pressure $[4,20]$. The full power of flow visualization, yet has to be transferred from the laboratory [22] to the swimming pool [4] but 
measurements of momentum and vorticity fluxes and power law relationships for different types of propulsion are under way. Two examples on hand propulsion and on vortex filament observations will be discussed next:

Figure 4 show some of the definitions used by Redondo and Cano (1979) to evaluate Forces produced by swimmers hands [20], in a recirculating water tank the drag coefficient of a hand model was seen to depend strongly on the local Reynolds number [18] as seen in figure 5, lift was also measured and compared with other authors [15, 16] but soon it was apparent that the unsteadiness of the process could only be quantified by looking at the fluid wakes. CFD has been used on an arm model as shown in figures 6-8 with an Open Foam Navier-Stokes solver, based in work by Petr Furmanek [23, 24], the patterns in plane hand flows, no-lift flow, can be seen for velocities of 2 and $4 \mathrm{~m} / \mathrm{s}$ in figures 7 and 8 . Simplifying sculling propulsion to lateral arm motions in a simple experiment such as depicted in figure 9, the underwater video bubble PIV analysis allows to estimate the plane velocity patterns as seen in a sequence in figure 10.
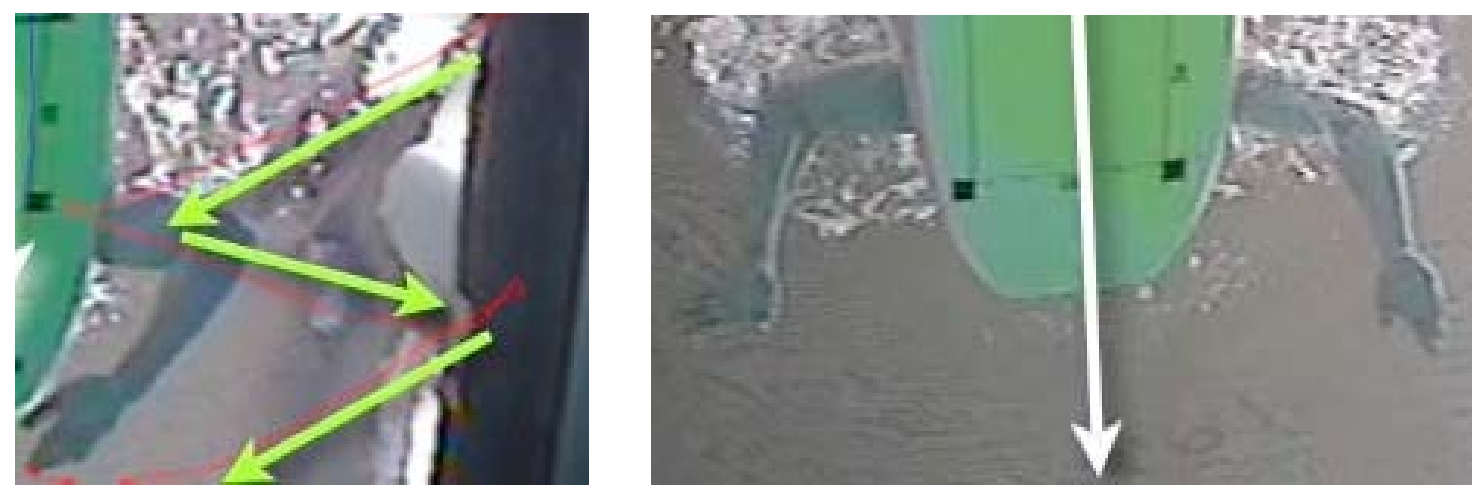

Figure 9. Description of a lateral sculling experiments, measuring propulsion only due to sculling on top of a floating board, air bubbles were also injected to visualize the wakes
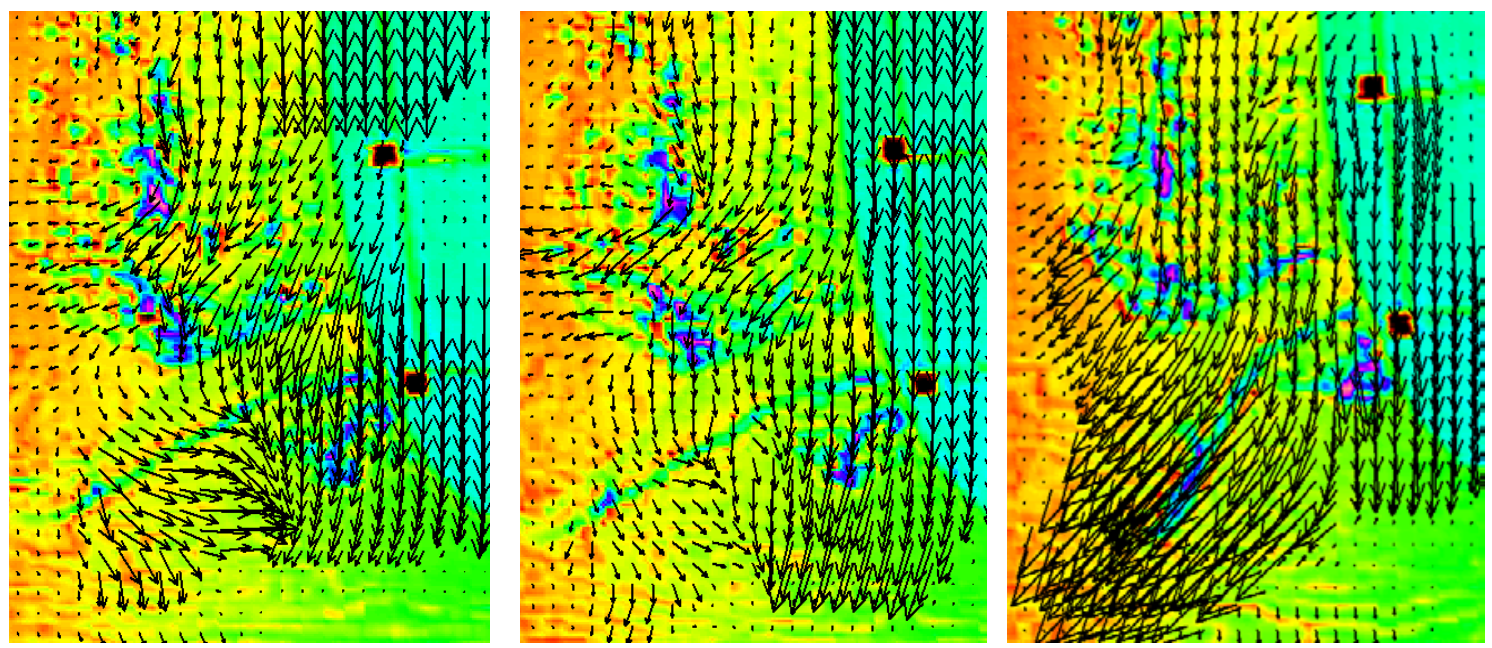

Figure 10. Evolution of the flow in a sculling motion 

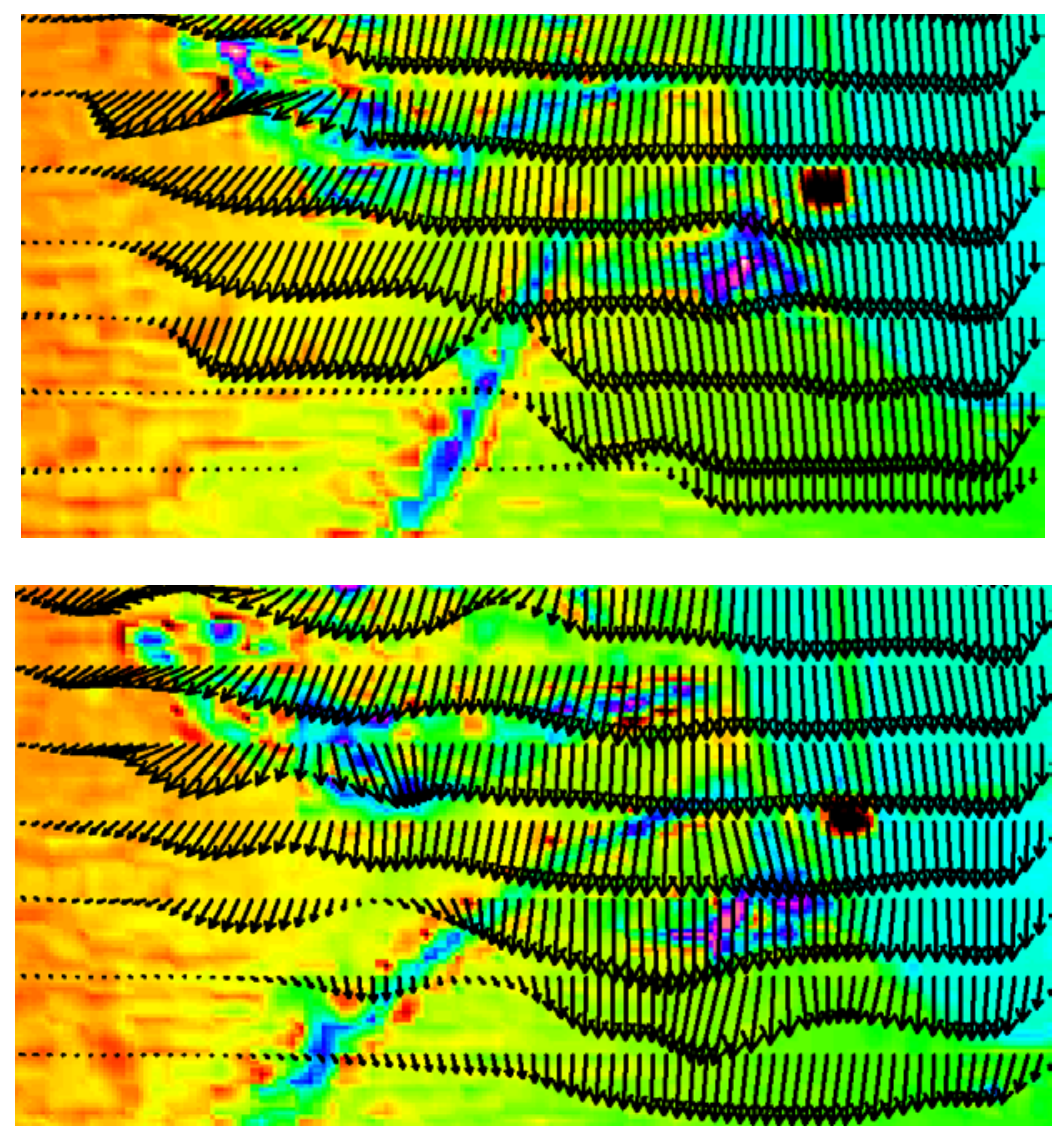

Figure 11. Forward velocity profiles produced by sculling, which allow the evaluation of momentum fluxes due to lateral hand motions.

To extend the measurements so best propulsive practices may be identified, maximum use of the available information is necessary. For the patterns of the velocity fields containing the full turbulent wake information random and vortical features such as seen in figures 10, 11 are projected down onto the plane of interest. This pattern is then viewed at an angle from the zenital position (typically $\sim 90^{\circ}$, selected for simplicity and persistence of the vertical vorticity as discussed in 2.1. Plane visualization planes with horizontal vorticity are also important [25-27]. An image of these velocity vectors is then used to calculate vorticity and horizontal stream functions and these are compared both before and after an specific propulsive event. In general the flow patterns will be moved and distorted, but unlike PIV vorticity patterns, or filaments will be quantified, the Local shear length scale, on the plane of visualization seems, together with the vorticity a good indicator. Helicity defined as the curl of velocity and vorticity is also important in a full 3D visualization, but precisely due to the experimental difficulty, both experiments and numerical simulations of comparable flows have to be performed side by side [27].

The flatness of the velocity or vorticity fields is an additional statistical descriptor that should be analyzed both in experiments and simulations, because it is strongly related to intermittency and non-steadiness, this parameter indicates the grade of planitud of the probability distribution functions (PDF, non-Gaussian) easily obtained from results represented above, and is defined from the structure functions (or velocity differences) as: 


$$
F=\frac{S_{4}}{\left(S_{2}\right)^{2}}
$$

With $\mathrm{S}_{4}$ the fourth order structure function and $\mathrm{S}_{2}$ the second order structure function (or Variance) As we have already commented, the flatness seems to be a very good indicator of the non-usual propulsive events, as when flatness changes with scale following a potential law, intermittency is present. Plotting in a logarithmic scale, the evolution of the flatness with the scale, filamentation may be compared with [27] and figures 15 below.

\section{Maximum Propulsion}

The unsteady propulsive movements including the changes in arms and feet sweepback angles are visible during competitive swimming due to accidental air entrainment, that thanks to the pressure low in the cores of strong vortices, tend to mark vortex filaments, these are easily visible during suddenly changes of the hand movement after changes of the hand movement direction is detached and it keeps rotating in the water during a short time, this type of flow detection as seen in figures 12, 13 and 16. The observation that only the best world-wide swimmers show consistently double vortex filaments and that theoretically (for Euler flow) a double filament improves propulsion points to a new hypothesis to be considered in statistical swimming experiments ( both numerical and experimental). As conclusions based on previous measurements where the difference in pressure between upper and lower hand have been measured during swimming, and compared with CFD simulations, it seems more important the distribution of velocity and vorticity in the wakes of the hands and feet than the pressure fields. New descriptors based on Vorticity patterns and Strouhal number are necessary

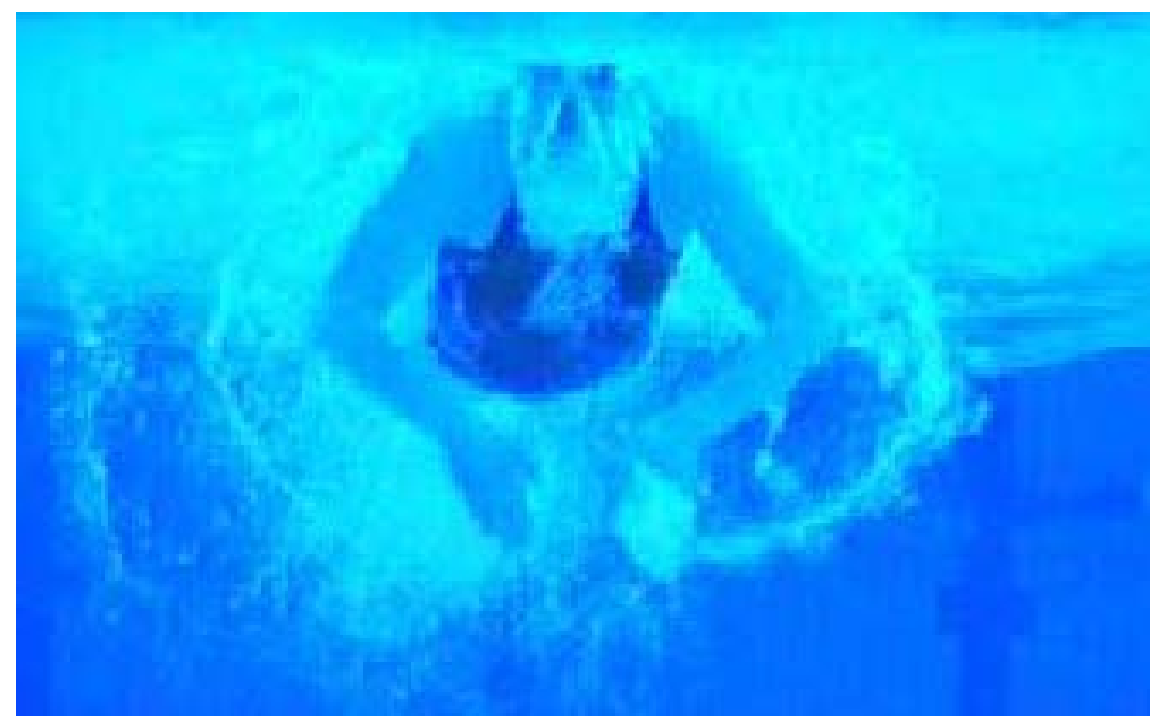

Figure 12. Symmetric vortex filament large structure in butterfly swimming 


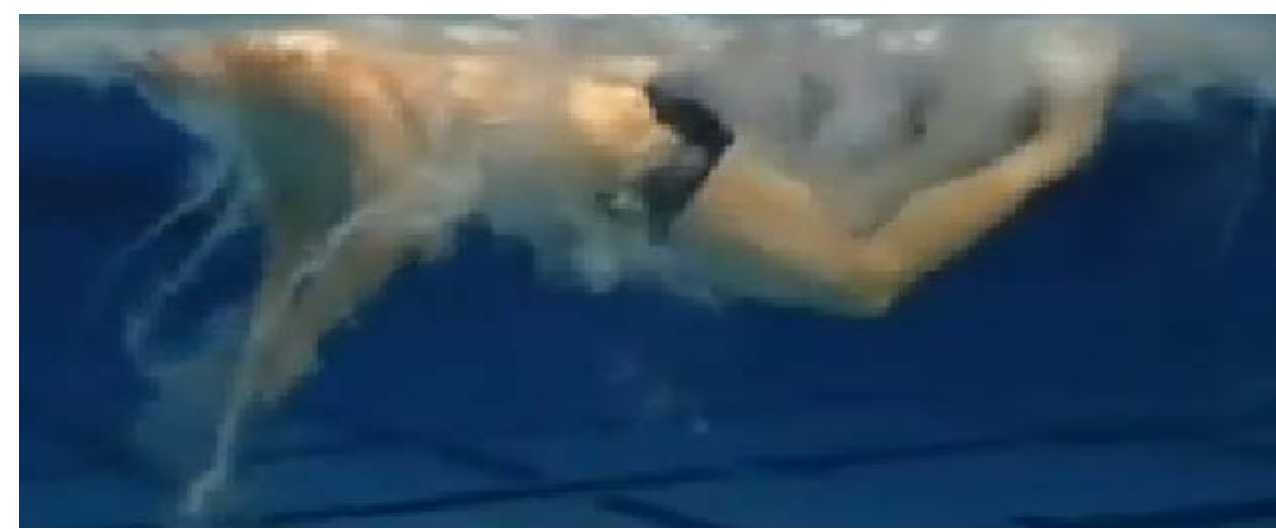

Figure 13. Detection of double vortex filaments produced in butterfly stroke by Michael Phelps, the visualization is random by accidental air bubble entrainment

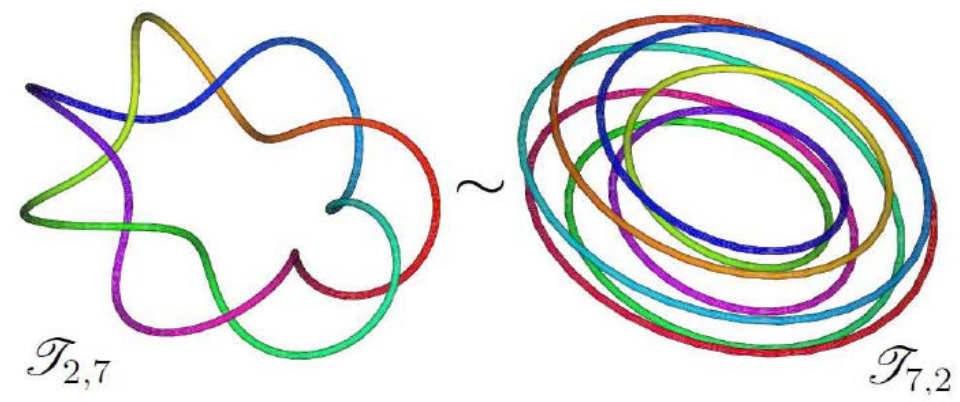

Figure 14. Different shapes of the topological equivalent vortex filaments [4, 25]
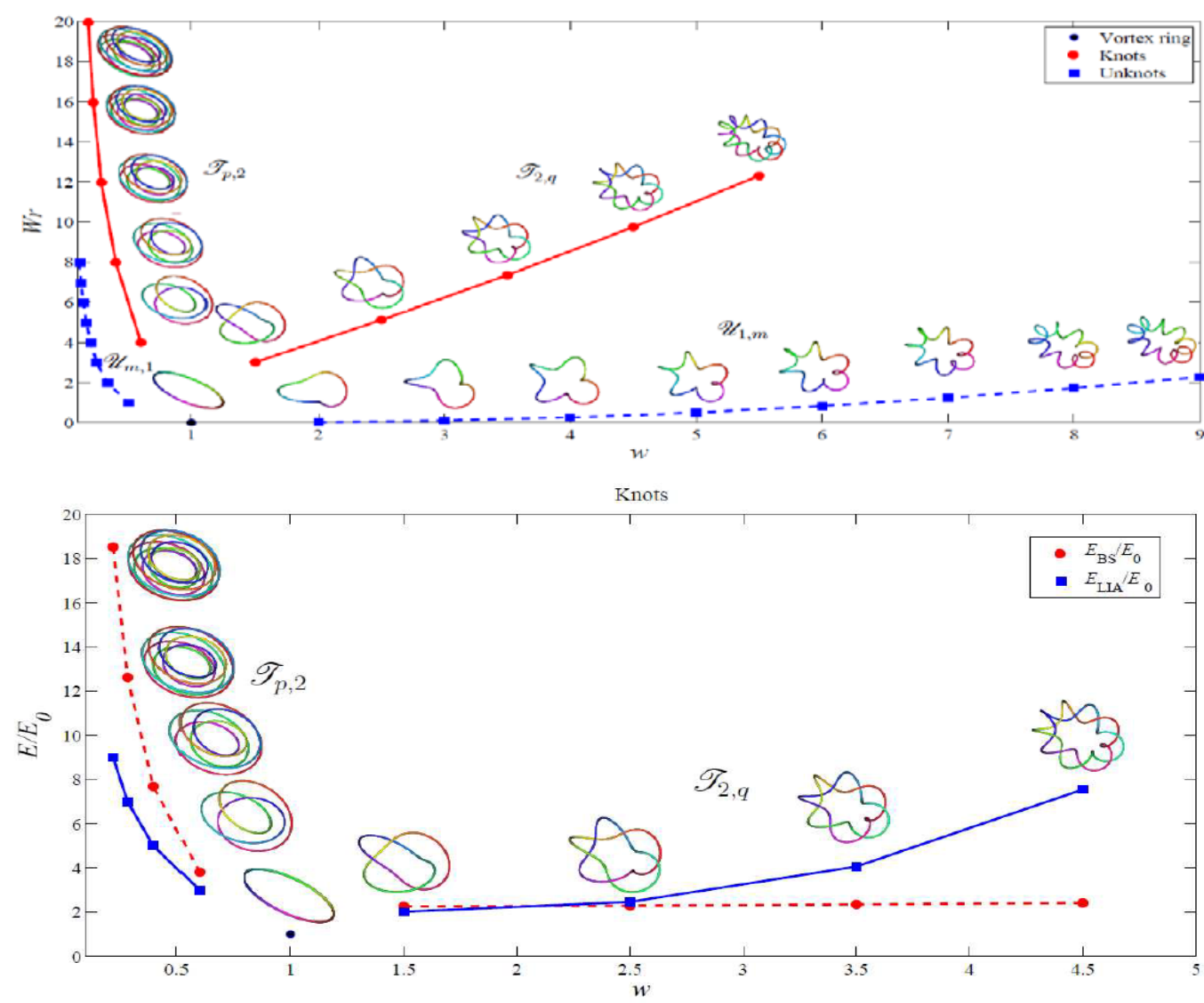

Figure 15. Variation of the velocity and energy for different winding numbers in a non-viscous flow [27]. 


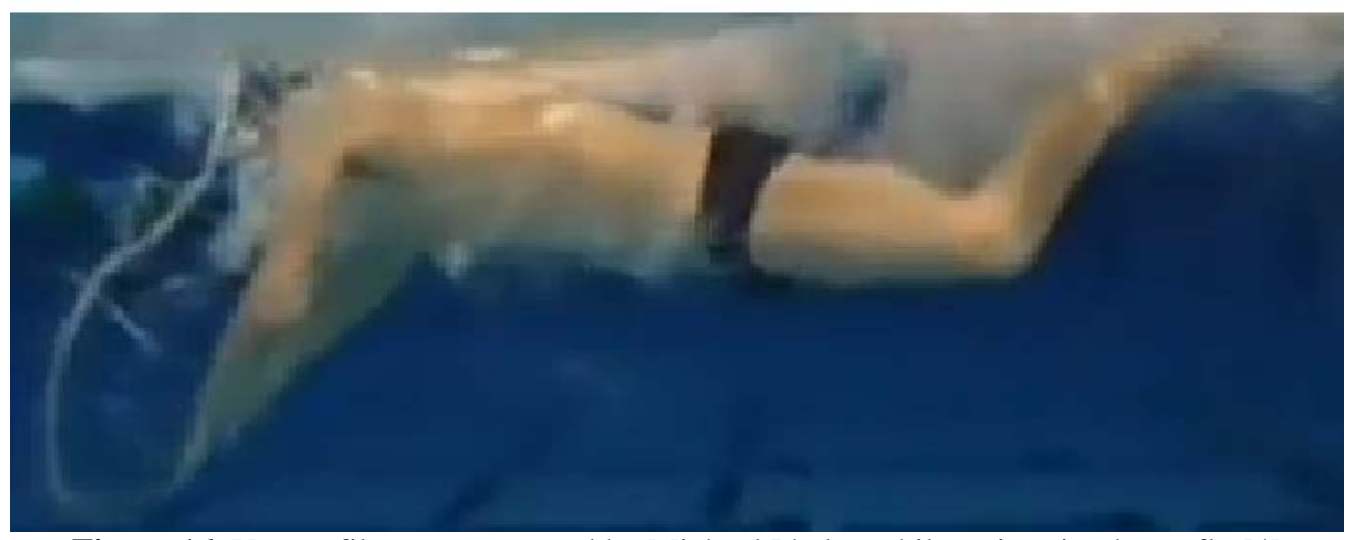

Figure 16. Vortex filament generated by Michael Phelps while swimming butterfly [4]

\section{Acknowledgements}

The financial support by the Spanish Ministry of Education and Science (projects. FTN2001-2220 DEP2006-56004 ) and Ministerio de Economia y Competividad DEP200908411 "ESTUDIO DE LOS MOVIMIENTOS PROPULSIVOS EN NATACION ("SCULLING")APLICANDO ANALISIS 3D, VISUALIZACION DE FLUIDOS, CFD Y PIV" is acknowledged.Thanks are also due to ERCOFTAC PELNoHT, (SIG 14) and the BSC (Barcelona Super Computer)..

\section{References}

[1] Castilla R., Sanchez M. A. and Redondo J. M. Vortical structures in stratified turbulent flows, in Turbulent Diffusion in the Environment, edited by Redondo J. M.and Babiano A. FRAGMA, Madrid. 1993: 113-120.

[2] Arellano, R., Pardillo, S., Gavilán. Underwater undulatory swimming: kinematic characteristics, vortex generation and application during the start, turn and Swimming Strokes. In: Proceedings of the XXth International Symposium on Biomechanics in Sports, Universidad de Granada. 2002.

[3] Arellano R., J.M. Terres-Nicol, J.M. Redondo. Fundamental hydrodynamics of swimming propulsion Portuguese Journal of Sport Sciences, 6. Sup. 1, 13. 2006.

[4] Redondo J.M., Arellano R., Carrillo A., Terres-Nicol J.M.: The role of Vorticity in Maximal Swimming Propulsion, Topical problems of Fluid Mechanics. CAS,: 47-54.2013.

[5] Bachlin, M., Troster, G. Swimming performance and technique evaluation with wearable acceleration sensors. Pervasive and Mobile Computing, 8,(1) 68-81.2012

[6] Jensen, U., J. V. Deschodt, L. M. Arsac, and A. H. Rouard, "Relative contribution of arms and legs in humans to propulsion in 25-m sprint front-crawl swimming," European Journal of Applied Physiology and Occupational Physiology, vol. 80, no. 3: 192-199, 1999.

[7] A. P. Hollander, G. de Groot, G. J. van Ingen Schenau, R. Kahman, and H. M. Toussaint, "Contribution of the legs in front crawl swimming," in Swimming Science V, B. E. Ungerechts, 1988.

[8] Reischle, K. and K. Wilke, Eds., pp. 39-43, Human Kinetics Publishers, Champaign, Ill, USA, 1988.

[9] Miller, D.I. Biomechanics of swimming, in Exercise and Sport Sciences Reviews, H.Willmore and J. F. Keogh, Eds.,pp. 219-248, Academic Press, New York, NY, USA, 1975. 
[10] Toussaint, H. and M. Truijens, "Biomechanical aspects of peak performance in human swimming,” Animal Biology, vol. 55, no.1: 17-40, 2005.

[11] Cantalapiedra I. R. and Redondo J. M., Mixing in Geophysical Flows, edited by Redondo J. M. and Metais O. (International Center for Numerical Methods, CIMNE 1995, 127.

[12] Redondo J. M. and Linden P. F., Geometrical observations of turbulent density interfaces, in The Mathematics of Deforming Surfaces, edited by Dritschel D. G. and Perkins R. J. (Clarendon Press Oxford) 1996, 221.

[13] Bixler, B. and Riewald, S. Analysis of a swimmer's hand and arm in steady flow conditions using computational fluid dynamics. Journal of Biomechanics, 35: 713717. 2002.

[14] Martin, B. Swimming: Forces on Aquatic Animals and Humans. In C. L. Vaughan (Ed.),Biomechanics of Sport (1 ed., pp. 35-51). Florida: CRC Press, Inc. 1989.

[15] Counsilman, J. E. The Application of Bernoulli's Principle to Human Propulsion in Water. Paper presented at the First International Symposium on "Biomechanics in Swimming, Water-Polo and Diving", Bruxelles. 1971.

[16] Maglischo, C.and Maglischo, E. Biomechanics of Aquatic Activities. In M. Adrian \& J. M. Cooper (Eds.), Biomechanics of Human Movement (2nd Ed.) 447470. Madison, Wisconsin: Brown \& Benchmark. 1995.

[17] Linden P.F. and Turner J.S. The formation of optimal vortex rings and the efficiency of propulsion devices. J. Fluid Mech, 427. 66-72. 2001.

[18] Redondo, J. M. Efecto de la Velocidad de la Brazada en el Coeficiente de Arrastre de las Manos. X Simposio de la Sociedad Ibérica de Biomecánica, Madrid. 1987

[19] Redondo, J. M., Arellano, R. Flow Visualization Using Reflective Particles in Analytical Movements of the Hand in Water: A Pilot Study. Barcelona: Escuela Técnica Superior de Canales y Puertos. UPC. Barcelona. 1998.

[20] Redondo, J. M., Cano, J. L. Primeras Determinaciones de los Efectos de Sustentación e Impulso en Natación. Natación, Saltos, Water-Polo, 1(5), 36-46. 1979.

[21] Redondo, J. M., Morris, S., Cano, J. L. Estudio sobre la Propulsión Producida por las Manos en Natación. Natación, Saltos, Water-Polo, 3(18), 32-37. 1981.

[22] Kazuo Matsuuchi and Yuki Muramatsu. Investigation of the Unsteady Mechanism in the Generation of Propulsive Force While Swimming Using a Synchronized Flow Visualization and Motion Analysis System, Biomechanics in Applications, Dr Vaclav Klika (Ed.). 2011.

[23] Dobeš, J., Fořt, J., Furst J., Furmánek P., Kladrubský, M., Kozel, K., Louda, P.: Numerical Solution of Transonical Flow around a Profile and a Wing II. Research report V-1850/05, VZLU a.s. 2005

[24] Furmanek, P. Numerical solution of Transonic flow past an Isolated profile, diploma work, 2004, CVUT FJFI, Praha. 2004.

[25] Maggioni F. Alamri S.Z, Barenghi C.F. and Ricca R.L.: Vortex knots dynamics in Euler fluids. Procedia IUTAM, Topological Fluid Dynamics II.

[26] Redondo, J.M.: Vortices and Coherent Structures in Swimming Wakes: Turbulent Flows, DAMTP. Cambridge University. F.E.N. report, 1990.

[27] Hochstein, S.; Blickhan, R. Vortex re-capturing and kinematics in human under water undulatory swimming. Human Movement Science, 30, 998-1007. 2011. 